\title{
ESTUDO DO MODELO DE CAMPO DE FASES PARA DIFERENTES ESPESSURAS DE INTERFACE*
}

Rodrigo Ramalho Maciel $^{1}$ Marcelo de Aquino Martorano

\section{Resumo}

O modelo de campo de fases de Wheeler, Boettinger e MacFadden (WBM), denominado modelo de interface aguda ("sharp"), e o modelo de Karma e Rappel (KR), denominado modelo de interface fina ("thin"), foram implementados no presente trabalho para estudar o efeito da espessura da interface sólido-líquido difusa na solidificação equiaxial de uma dendrita de metal puro. As equações destes modelos foram adimensionalizadas e solucionadas pelo método das diferenças finitas, na sua formulação explícita. O domínio de cálculo, que foi assumido igual a um quarto do domínio real utilizando a simetria do problema, foi suficientemente grande para ser considerado semi-infinito. As imagens das dendritas calculadas pelo modelo WBM e as curvas de velocidade da ponta dendrítica em função do tempo não convergem, ou seja, sofrem alterações para reduções de espessura até cerca de duas vezes o comprimento de capilaridade. No entanto, os resultados do modelo $\mathrm{KR}$ convergem para espessuras menores ou iguais a quatro vezes o comprimento de capilaridade. A velocidade de estado-estacionário calculada pelo modelo $\mathrm{KR}$ converge para o valor obtido em uma solução do modelo clássico de solidificação na faixa de espessuras estudadas, ao passo que o valor fornecido pelo modelo WBM distancia-se cada vez mais do valor estacionário conforme a espessura da interface decresce.

Palavras-chave: Modelo de Campo de Fases; Interface Aguda; Interface fina.

\section{STUDY ON PHASE FIELD MODEL FOR DIFFERENT INTERFACE THICKNESS Abstract}

The phase-field model proposed by Wheeler, Boettinger, and MacFadden (WBM), denoted as the sharp interface model, and the model proposed by Karma and Rappel (KR), referred as the thin interface model, were implemented to investigate the effects of the thickness of the diffuse solid-liquid interface on the growth of an equiaxed dendrite of a pure metal. The dimensionless form of the model equations were solved using an explicit finite difference method. One quarter of the equiaxed dendrite was simulated owing to the symmetry of the problem and the calculation domain was large enough to be considered as semi-infinite. The morphology of the dendrite images and the curves of dendrite tip velocity as a function of time calculated with the WBM model do not converge for a decreasing interface thickness down to a value two times as large as the capillarity length. However, KR model results converge for a thickness equal to or lower than four times the capillarity length. The stationary velocity of the KR model converges to the solution of the classical model of solidification in the range of interface thicknesses considered. On the other hand, the stationary velocity calculated with the WBM model diverges from the classical model as the interface thickness decreases.

Keywords: Phase-Field Method; Sharp interface; Thin Interface.

Mestrando, estudante, Dep. De Eng. Metalúrgica e de Materiais, USP, São Paulo, SP, Brasil.

2 Dr., Professor, Dep. De Eng. Metalúrgica e de Materiais, USP, São Paulo, SP, Brasil. 


\section{INTRODUÇÃO}

Os processos de solidificação de metais e suas ligas apresentam elevada importância na indústria metalúrgica. A movimentação da interface sólido-líquido define a formação de dendritas e outras morfologias da fase sólida durante a solidificação. Existem modelos matemáticos para previsão da formação de dendritas tanto em metais puros [1] como em ligas [2]. Em uma escala de tamanho microscópica, o modelo matemático clássico para a solidificação de um metal puro é dado pelas equações abaixo:

$$
\begin{gathered}
\frac{\partial T}{\partial t}=\alpha \nabla^{2} T \\
\kappa_{S}\left(\frac{\partial T}{\partial n}\right)_{S}-\kappa_{L}\left(\frac{\partial T}{\partial n}\right)_{L}=L V_{n} \\
T=T_{m}-\frac{T_{m} \gamma}{L}\left(\frac{1}{R_{1}}+\frac{1}{R_{2}}\right)-\frac{V_{n}}{\mu}
\end{gathered}
$$

onde $T$ representa a temperatura, $t$ o tempo, $\alpha$ a difusividade térmica, $\kappa_{L}$ e $\kappa_{S}$ a condutividade térmica do líquido e do sólido, respectivamente, $L$ o calor latente por unidade de volume, $\gamma$ a energia de interface por unidade de área, $T_{m}$ a temperatura de fusão, $R_{1}$ e $R_{2}$ são os raios de curvatura principais ao longo da interface, $\mu$ é o coeficiente cinético e $V_{n}$ a velocidade da interface na sua direção normal [3]. Neste modelo, a interface sólido-líquido é assumida ser uma superfície matemática ideal, ou seja, sem espessura, e deve ser acompanhada em detalhes quando as equações do modelo clássico são solucionadas.

O modelo do campo de fases ("phase field") é uma possível alternativa ao modelo clássico e possui diversas vantagens em muitas situações [4]. Nesse modelo, a interface sólido-líquido é chamada de difusa e consiste de uma região que possui uma espessura finita. Um mesmo conjunto de equações é solucionado no sólido, no líquido e na região da interface, portanto, não são necessárias técnicas especiais para o acompanhamento da interface e nem a aplicação de condições de contorno sobre a mesma. Neste modelo, define-se a variável de fase, $\phi$, que assume diferentes valores no sólido, no líquido e na região da interface. A construção de diversos modelos de campo de fases começa com a definição do seguinte funcional para a energia livre total $(F)$ de um sistema de metal puro contendo fase líquida e ou fase sólida:

$$
F=\int_{V}\left[\frac{\varepsilon^{2}}{2}|\nabla \phi|^{2}-L \frac{T_{m}-T}{T_{m}} p(\phi)+W g(\phi)\right] d V
$$

onde $V$ é o volume do sistema, $g(\phi)$ é uma função que auxilia na criação de uma barreira energética entre as fases sólido e líquido, sendo que $W$ é o máximo desta barreira, $p(\phi)$ é uma função para interpolar a energia livre na região da interface difusa e $\varepsilon$ é o coeficiente do termo que representa a chamada energia gradiente [3]. $O$ coeficiente $\varepsilon$ é usualmente descrito como função da orientação $\theta$ do vetor $\vec{\nabla} \phi$ a partir da relação $\varepsilon(\theta)=\varepsilon_{o} \eta(\theta)$, onde $\varepsilon_{o}$ é uma constante. A orientação $\theta$, que é calculada como $\theta=\tan ^{-1}\left(\frac{\partial \phi / \partial y}{\partial \phi / \partial x}\right)$, coincide com a orientação da interface difusa quando calculado no interior da região desta interface. A equação para evolução da variável de fase foi postulada como sendo $\frac{1}{M} \frac{\partial \phi}{\partial t}=-\frac{\delta F}{\delta \phi}$, onde $M$ é a mobilidade e $\frac{\delta F}{\delta \phi}$ é 
a derivada variacional de $F$ em relação ao campo da variável de fase, $\phi$. Substituindo a Eq. (4) nesta equação e definindo $g(\phi)=-\frac{\phi^{2}}{2}+\frac{\phi^{4}}{4}$ e $p(\phi)=\phi-$ $\frac{2}{3} \phi^{3}+\frac{\phi^{5}}{5}$, obtém-se

$$
\frac{1}{M} \frac{\partial \phi}{\partial t}=\varepsilon_{o}^{2} \nabla_{a}^{2} \phi+L \frac{T_{m}-T}{T_{m}}\left(1-\phi^{2}\right)^{2}-W \phi\left(1-\phi^{2}\right)
$$

onde

$$
\begin{aligned}
\nabla_{a}^{2} \phi=\eta \eta^{\prime}(2 & \left.\cos 2 \theta \phi_{x y}+\sin 2 \theta\left(\phi_{y y}-\phi_{x x}\right)\right)+\eta^{2} \nabla^{2} \phi \\
+ & \frac{1}{2}\left(\eta \eta^{\prime}\right)^{\prime}\left(\nabla^{2} \phi+\cos 2 \theta\left(\phi_{y y}-\phi_{x x}\right)-2 \sin 2 \theta \phi_{x y}\right)
\end{aligned}
$$

sendo $\eta^{\prime}=\frac{d \eta}{d \theta}$. A espessura da região da interface difusa, $\delta_{0}$, é assumida ser a distância onde $\phi$ varia entre 0 e 1. Esta espessura está relacionada com os parâmetros da Eq. (5) por

$$
\delta_{0}=\frac{\varepsilon}{\sqrt{W}} .
$$

A equação para evolução da variável de fase empregada em diversos modelos propostos na literatura pode ser ajustada no formato das Eqs. (5) e (6). Foi observado que é possível fazer com que os resultados destas equações reproduzam quantitativamente os resultados do modelo clássico, dado pelas Eqs. (1) a (3). Para isto, é necessário estabelecer relações entre os parâmetros das Eqs. (5) e (6) e os parâmetros físicos das Eqs. (1) a (3). Wheeler, Boettinger e MacFadden [2] propuseram as seguintes relações

$$
\begin{gathered}
\frac{1}{\mu}=a_{1} \frac{T_{m} \sqrt{W}}{M L \varepsilon} \\
\gamma=a_{1} \varepsilon \sqrt{W}
\end{gathered}
$$

onde a 1 uma constante igual a $1 / 6 \sqrt{2}$. Karma e Rappel [1] mostraram a partir de uma análise assintótica rigorosa que a Eq. (8) é válida apenas quando a espessura $\delta_{0}$ é suficientemente fina para que as variações de temperatura através da região da interface sejam desprezíveis. Esta espessura deve ser muito menor que a menor escala de comprimento do problema clássico, dada pelo comprimento de capilaridade $d_{0}=\frac{\gamma C_{p} T_{m}}{L^{2}}$. Quando as espessuras são maiores e as variações de temperatura através da interface não podem ser desprezadas, Karma e Rappel [1] mostraram que um termo deve ser adicionado à Eq. (8), resultando em

$$
\frac{1}{\mu}=a_{1}\left[\frac{T_{m} \sqrt{W}}{M L \varepsilon}+a_{2} \frac{\varepsilon L}{\sqrt{W} \alpha c_{p}}\right]
$$

onde $\mathrm{a}_{2}$ é uma constante dependente das funções $g(\phi)$ e $p(\phi), \alpha$ é a difusividade térmica e $c_{p}$ é o calor específico. Utilizando a Eq. Erro! Fonte de referência não encontrada., foi possível relacionar os parâmetros do modelo do campo de fases e do modelo clássico para maiores valores de espessura $\delta_{0}$, permitindo a utilização de malhas numéricas com menor número de elementos para obtenção da solução numérica. Uma segunda importante utilidade da Eq. (10) é a eliminação do superresfriamento cinético, desprezível para a maior parte dos metais, impondo-se $1 / \mu=$ 0 . Nas relações apresentadas, obtidas pela análise assintótica do modelo do campo de fases, diversos termos foram assumidos desprezíveis, o que ocorre quando a 
espessura da interface, $\delta_{0}$, é suficientemente reduzida. Logo, os resultados calculados com espessuras maiores podem causar discrepâncias significativas em relação ao modelo clássico.

O efeito da espessura da interface difusa, $\delta_{0}$, sobre os resultados do modelo do campo de fases é, portanto, um tópico de pesquisa de grande interesse. A possibilidade de se utilizar espessuras maiores e ainda obter resultados quantitativamente precisos tem grande impacto na viabilidade e custo das simulações [1]. Portanto, este trabalho tem como objetivo estudar quantitativamente o efeito da variação da espessura da interface difusa sobre os resultados de dois tipos de modelos de campo de fases: o modelo de Wheeler, Boettinger e MacFadden (WBM) [2], chamado de modelo de interface aguda ("sharp"), e o modelo de Karma e Rappel (KR) [1], conhecido como modelo de interface fina ("thin"). Estes dois modelos podem ser resumidos pelas Eqs. (5) e (6), porém o modelo WBM utiliza a Eq. (8) para calcular $M$ a partir da definição do coeficiente cinético, $\mu$, enquanto no modelo $\mathrm{KR}$ o valor de $M$ é calculado a partir da Eq. (10), impondo-se $1 / \mu=0$. Os resultados destes dois modelos, aplicados à simulação da solidificação de uma dendrita equiaxial de metal puro, serão avaliados e comparados. Serão analisadas qualitativamente a morfologia das estruturas dendríticas simuladas e quantitativamente a velocidade de crescimento da ponta dendrítica.

\section{MATERIAIS E MÉTODOS}

Foi implementado um modelo bidimensional de campo de fases que se reduz aos modelos WBM ou KR mediante modificação de alguns poucos parâmetros. Neste modelo, a variável de fase assume $\phi=1$ no sólido, $\phi=-1$ no líquido e $-1<\phi$ $<1$ na região da interface. A função de orientação $\eta(\theta)$ do coeficiente $\varepsilon$ foi definida como $\eta=1+s \cos 4 \theta$, sendo $s=0,05$. Devido à relação entre $\varepsilon$ e a energia interfacial $\gamma$ dada pela Eq. (9), a função $\eta(\theta)$ causa propositalmente uma anisotropia em $\gamma$, que apresentará pontos de mínimo em quatro direções ortogonais para simulações bidimensionais. Finalmente, as Eqs. (5) e (6) foram adimensionalizadas, resultando em

$$
\begin{gathered}
\frac{1}{M^{*}} \frac{\partial \phi}{\partial t^{*}}=\varepsilon_{o}^{* 2} \nabla_{a}^{* 2} \phi-a_{1} \varepsilon_{o}^{*} T^{*}\left(1-\phi^{2}\right)^{2}+\phi\left(1-\phi^{2}\right) \\
\nabla_{\mathrm{a}}^{*^{2}} \phi=\eta \eta^{\prime}\left[2 \cos 2 \theta \phi_{x^{*} y^{*}}+\sin 2 \theta\left(\phi_{y^{*} y^{*}}-\phi_{x^{*} x^{*}}\right)\right]+\eta^{2} \nabla^{* 2} \phi \\
+\frac{1}{2}\left(\eta \eta^{\prime}\right)^{\prime}\left[\nabla^{* 2} \phi+\cos 2 \theta\left(\phi_{y^{*} y^{*}}-\phi_{x^{*} x^{*}}\right)-2 \sin 2 \theta \phi_{x^{*} y^{*}}\right]
\end{gathered}
$$

a partir da definição das seguintes variáveis adimensionais

$$
\begin{gathered}
T^{*}=\left(T-T_{m}\right) \frac{C_{p}}{L} \\
x^{*}=\frac{x}{d_{o}}, y^{*}=\frac{y}{d_{o}} \\
t^{*}=t \frac{\alpha}{d_{o}^{2}}
\end{gathered}
$$

onde $d_{0}=\frac{a_{1} \varepsilon_{0} \sqrt{W} C_{p} T_{m}}{L^{2}}$ que, devido à Eq. (9), equivale ao comprimento de capilaridade definido anteriormente. No modelo $\mathrm{KR}$, o produto $a_{1} \varepsilon_{o}^{*}$ foi denominado $\lambda$. No formato 
adimensional dado pela Eq. (11), restaram apenas os dois parâmetros adimensionais apresentados abaixo:

$$
\begin{aligned}
\varepsilon_{o}^{*} & =\frac{\varepsilon_{o}}{d_{o} \sqrt{W}} \\
M^{*} & =\frac{M W d_{o}^{2}}{\alpha}
\end{aligned}
$$

onde, devido à Eq. (7), $\varepsilon_{o}^{*}$ representa a espessura da interface adimensionalizada em relação ao comprimento de capilaridade $\left(d_{0}\right)$ e $M^{*}$ é a mobilidade adimensional.

A equação de condução de calor, apresentada abaixo no formato adimensional, também foi solucionada em conjunto com a Eq. (11)

$$
\frac{\partial T^{*}}{\partial t^{*}}=\nabla^{* 2} T^{*}+\frac{1}{2} \frac{\partial \phi}{\partial t^{*}}
$$

A mobilidade $M^{*}$ no modelo WBM e no modelo KR foram calculadas utilizando a forma adimensional das Eqs. (8) e (10), respectivamente,Erro! Fonte de referência não encontrada. e impondo $1 / \mu=0$ no caso do modelo $K R$, resultando em

$$
\begin{gathered}
\frac{1}{M^{*}}=\frac{\varepsilon_{0}^{* 2} \eta}{\mu^{*}}(\mathrm{WBM}) \\
\frac{1}{M^{*}}=a_{1} a_{2} \varepsilon_{0}^{* 3} \eta^{2}(\mathrm{KR})
\end{gathered}
$$

onde $\mu^{*}=\mu L d_{0} / C_{P} \alpha$ é o coeficiente cinético adimensional e as constantes $a_{1}=$ 0,8839 e $a_{2}=0,6267$ foram propostas por Karma e Rappel [1].

As Eqs. (11) e (12) foram discretizadas utilizando-se a formulação explícita do método das diferenças finitas, com espaçamento de malha igual a $\Delta x^{*}=0.4 \varepsilon_{o}^{*}$, garantindo aproximadamente 10 nós da malha numérica na região da interface, e com passo de tempo $\Delta t^{*}=\frac{\Delta x^{* 2}}{10}$. Foi utilizado um domínio de cálculo retangular de dimensões 1500 por 1500 comprimentos de capilaridade e no seu contorno, na direção perpendicular, foram aplicadas condições de gradiente nulo para $\phi$ e $T$. Em todas as simulações do modelo WBM, adotou-se $\mu^{*}=1.18$, que resulta em um valor de mobilidade $M^{*}$ (Eq. (19)) igual àquele utilizado no modelo KR para a espessura $\varepsilon_{o}^{*}=10$. No entanto, para diferentes espessuras, os valores de $M^{*}$ do modelo WBM serão alterados, já que $\mu^{*}$ foi mantido constante. Como condição inicial, foi posicionado um quarto de um círculo sólido $(\phi=1)$ de raio $5 d_{0}$ no canto inferior esquerdo do domínio, onde a temperatura inicial, $\Delta$, foi assumida uniforme $T^{*}=\Delta=$ $-0,55$. Note que os únicos parâmetros de entrada para os modelos serão $\varepsilon_{0}^{*}$ e $\Delta$, que é o super-resfriamento inicial. Utilizando a simetria do problema, foi apenas simulado um quarto de uma dendrita equiaxial em crescimento no interior de um líquido superresfriado. $\mathrm{Na}$ apresentação das imagens das dendritas simuladas, o domínio de cálculo foi repetido e combinado para mostrar a imagem de uma dendrita equiaxial completa.

\section{RESULTADOS E DISCUSSÃO}

As imagens das dendritas equiaxiais calculadas pelo modelo WBM (interface aguda) e KR (interface fina) estão apresentadas na Figura 1 e Figura 2, respectivamente. Em cada imagem, as linhas representam curvas de nível para $\phi=$ 0 , indicando a posição da interface em diferentes instantes de tempo e mostrando a evolução morfológica das dendritas. A imagem no quadrante superior direito mostra 
o campo de temperaturas no instante $t^{*}=5 \times 10^{4}$, indicando um líquido superresfriado em relação ao sólido, como esperado para o crescimento equiaxial. Notase que o crescimento ocorre nas quatro direções de menor energia interfacial, impostas pela função de anisotropia $\eta(\theta)$, como esperado no crescimento dendrítico de grande parte dos metais. O conjunto de imagens apresentadas da Figura 1(a) até a Figura 1(e) mostra o efeito da redução da espessura da interface difusa, $\varepsilon_{0}^{*}$, na morfologia dendrítica. Quando $\varepsilon_{0}^{*}$ é reduzido de 10 até 2, ocorrem variações nos detalhes da morfologia. Nos resultados do modelo WBM, nota-se que para uma redução de espessura $\left(\frac{\varepsilon}{\sqrt{W}}\right)$ de 4 para 2 vezes o comprimento de capilaridade $\left(d_{0}\right)$ ainda aparecem diferenças, indicando que a convergência em direção ao modelo clássico ainda não ocorreu. No caso do modelo WBM, esta convergência é esperada somente quando $\varepsilon_{0}^{*}<<1$, ou seja, quando a espessura da interface for muito menor do que o comprimento de capilaridade.

As imagens de dendritas para o modelo $\mathrm{KR}$ (interface fina) estão apresentadas na Figura 2 e mostram diferenças praticamente imperceptíveis na morfologia dendrítica e no campo de temperaturas quando $\varepsilon_{0}^{*}$ é alterado de 4 para 2. Desta forma, conclui-se que este modelo convergiu para o modelo clássico com valores de espessura de 4 vezes o comprimento de capilaridade e, portanto, com $\varepsilon_{0}^{*}$ $>1$, que representa uma condição mais eficiente em comparação à condição do modelo WBM $\left(\varepsilon_{0}^{*}<<1\right)$. A Figura 3 apresenta o perfil da variável de fase através da interface na região da ponta dendrítica para o caso do modelo KR. Cada símbolo das curvas indica a posição de um nó da malha numérica, mostrando que existem pelo menos 10 pontos, que é uma quantidade maior do que a usualmente sugerida para representar corretamente as variações da variável de fase através da região da interface. 


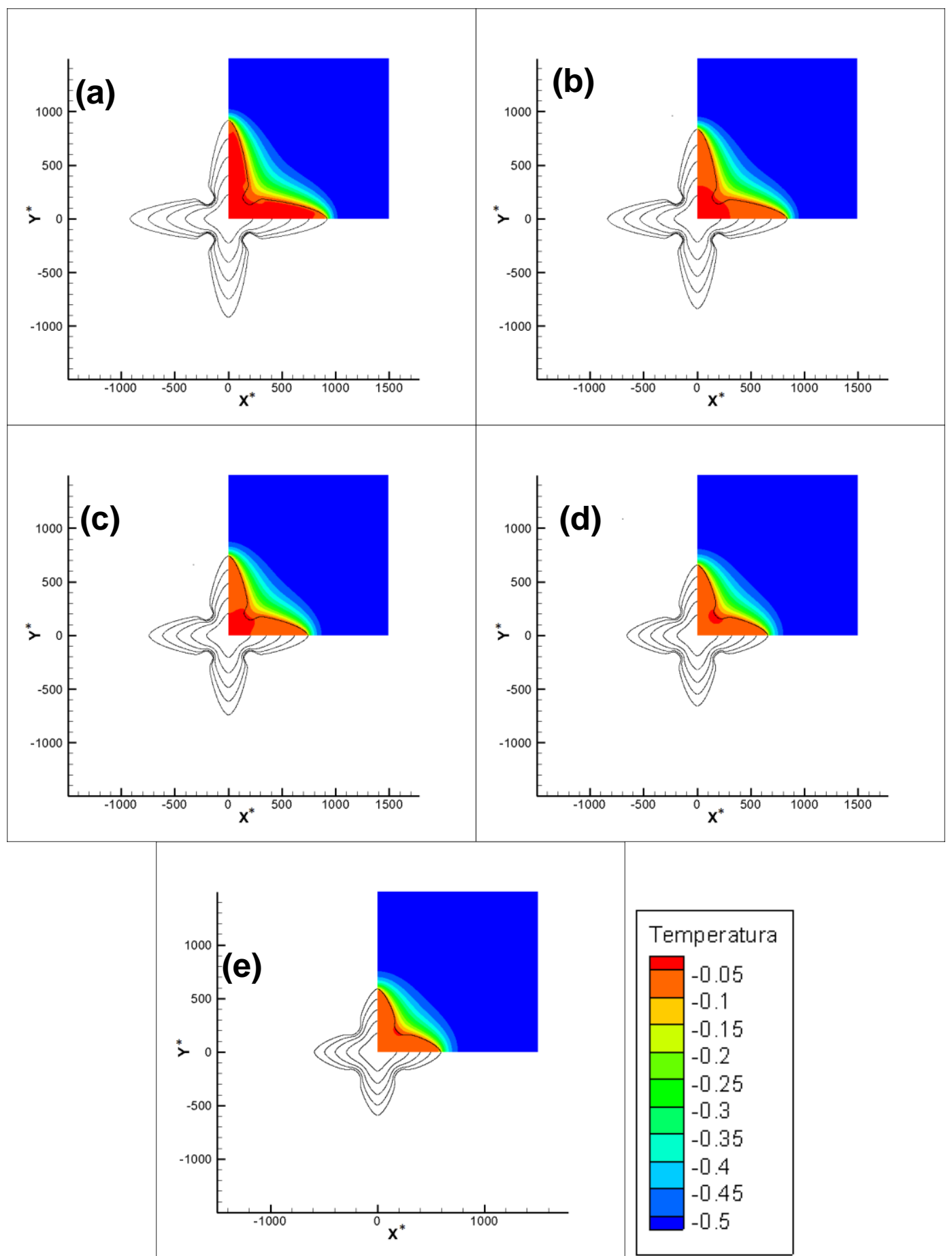

Figura 1 - Imagens calculadas das dendritas equiaxiais para o modelo WBM (interface aguda) utilizando as seguintes espessuras de interface difusa: (a) $\varepsilon_{o}^{*}=10$; (b) $\varepsilon_{o}^{*}=8$; (c) $\varepsilon_{o}^{*}=6$; (d) $\varepsilon_{o}^{*}=4$; (e) $\varepsilon_{o}^{*}=2$. As linhas são curvas de nível para $\phi=0$ nos instantes $t^{*}=1 \times 10^{4}, 2 \times 10^{4}, 3 \times 10^{4}, 4 \times 10^{4}$ e $5 \times 10^{4}$. A imagem no quadrante superior direito mostra o campo $T^{*}$ em $t^{*}=5 \times 10^{4}(\Delta=-0,55, s=0,05)$. 



Figura 2 - Imagens calculadas das dendritas equiaxiais para o modelo KR (interface fina) utilizando as seguintes espessuras de interface difusa: (a) $\varepsilon_{o}^{*}=10$; (b) $\varepsilon_{o}^{*}=8$;

(c) $\varepsilon_{o}^{*}=6$; (d) $\varepsilon_{o}^{*}=4$; (e) $\varepsilon_{o}^{*}=2$. As linhas são curvas de nível para $\phi=0$ nos instantes $t^{*}=1 \times 10^{4}, 2 \times 10^{4}, 3 \times 10^{4}, 4 \times 10^{4}$ e $5 \times 10^{4}$. A imagem no quadrante superior direito mostra o campo $T^{*}$ em $t^{*}=5 \times 10^{4}(\Delta=-0,55, s=0,05)$.

A velocidade de crescimento da ponta dendrítica, $V$, definida de forma adimensional como $V^{*}=\frac{V d_{0}}{\alpha}$, foi calculada e está apresentada na Figura 44 para os dois modelos examinados. Em todas as curvas, nota-se que a velocidade é inicialmente elevada e tende a um valor constante, indicando que o estadoestacionário foi atingido, como esperado para o crescimento dendrítico equiaxial em 
um líquido semi-infinito. Também está mostrada na figura, a velocidade da ponta em estado estacionário calculada pelo modelo clássico para $\frac{1}{\mu}=0$, solucionado pelo método de Green [1]. Observa-se que as curvas de velocidade calculadas pelo modelo WBM (Figura 4(a)) sempre apresentaram alterações significativas para cada redução de $\varepsilon_{0}^{*}$, indicando que a convergência não está sendo atingida. Os resultados mostram ainda que a velocidade de estado-estacionário obtida por este modelo distancia-se cada vez mais do modelo clássico com a redução de $\varepsilon_{0}^{*}$. Este distanciamento é provavelmente causado pelo super-resfriamento cinético, que não se pode eliminar do modelo WBM, mas que não existe na solução apresentada para o modelo clássico. Por outro lado, os resultados do modelo KR (Figura 4(b)) mostram que as curvas tanto para a região transiente como estacionária convergem para uma espessura $\varepsilon_{0}^{*} \leq 4$, como também observado qualitativamente nas imagens de dendrita calculadas. No estado-estacionário elas se aproximam da solução do modelo clássico, mostrando que o super-resfriamento cinético foi realmente eliminado $e$ indicando a melhor eficiência do modelo de interface fina em comparação ao de interface aguda. Os resultados calculados por Karma e Rappel [1] tanto na região transiente como estacionária para $\varepsilon_{0}^{*}=7,19$ também estão apresentados na Figura 4(a) e mostram boa concordância com os resultados do presente trabalho para $\varepsilon_{0}^{*}=8$.

À medida que a espessura da interface é reduzida, a Figura 5(a) e (b) mostra mais claramente a divergência do modelo WBM (interface aguda) e a convergência do modelo KR (interface fina) para a velocidade de crescimento em regime estacionário dada pelo modelo clássico. Os cálculos de Karma e Rappel [1] também estão apresentados na Figura 5(a) e, analogamente aos resultados do presente trabalho, também mostram uma convergência do modelo KR para $\varepsilon_{0}^{*}$ entre 4 e 6.

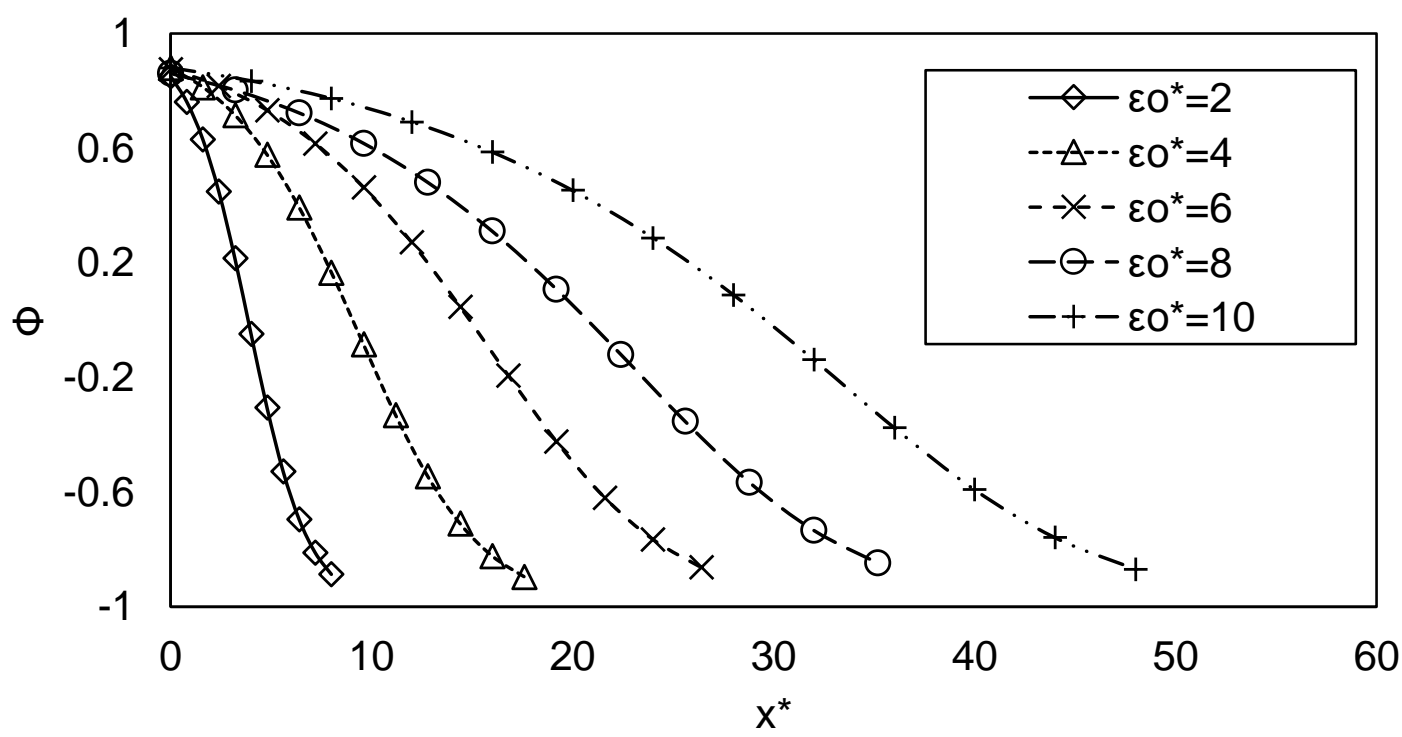

Figura 3 - Perfil da variável de fase através da interface calculado pelo modelo KR (interface fina) para as diferentes espessuras de interface $\left(\varepsilon_{0}^{*}\right)$, sendo que a posição de cada nó da malha numérica está indicada pelos símbolos. 

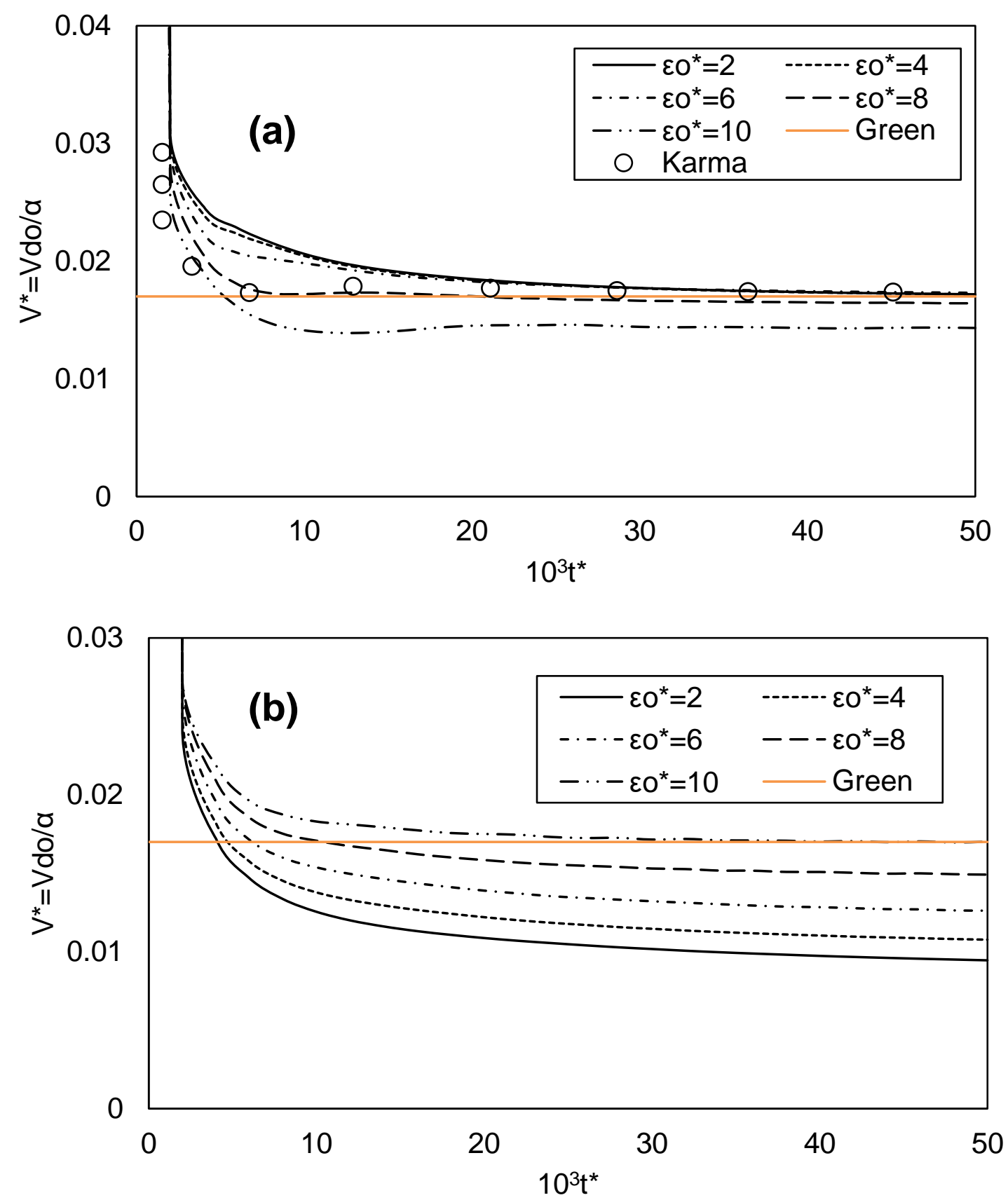

Figura 4 - Velocidade adimensional de crescimento da ponta dendrítica em função do tempo para diferentes espessuras $\varepsilon_{0}^{*}$ para os modelos: (a) WBM (interface aguda) e (b) KR (interface fina). A curva de velocidade obtida pelo modelo clássico (Green) para o crescimento em estado estacionário também está mostrada. A solução numérica obtida por Karma e Rappel [1] está apresentada em (a). ( $\Delta=$

$$
-0,55, s=0,05)
$$



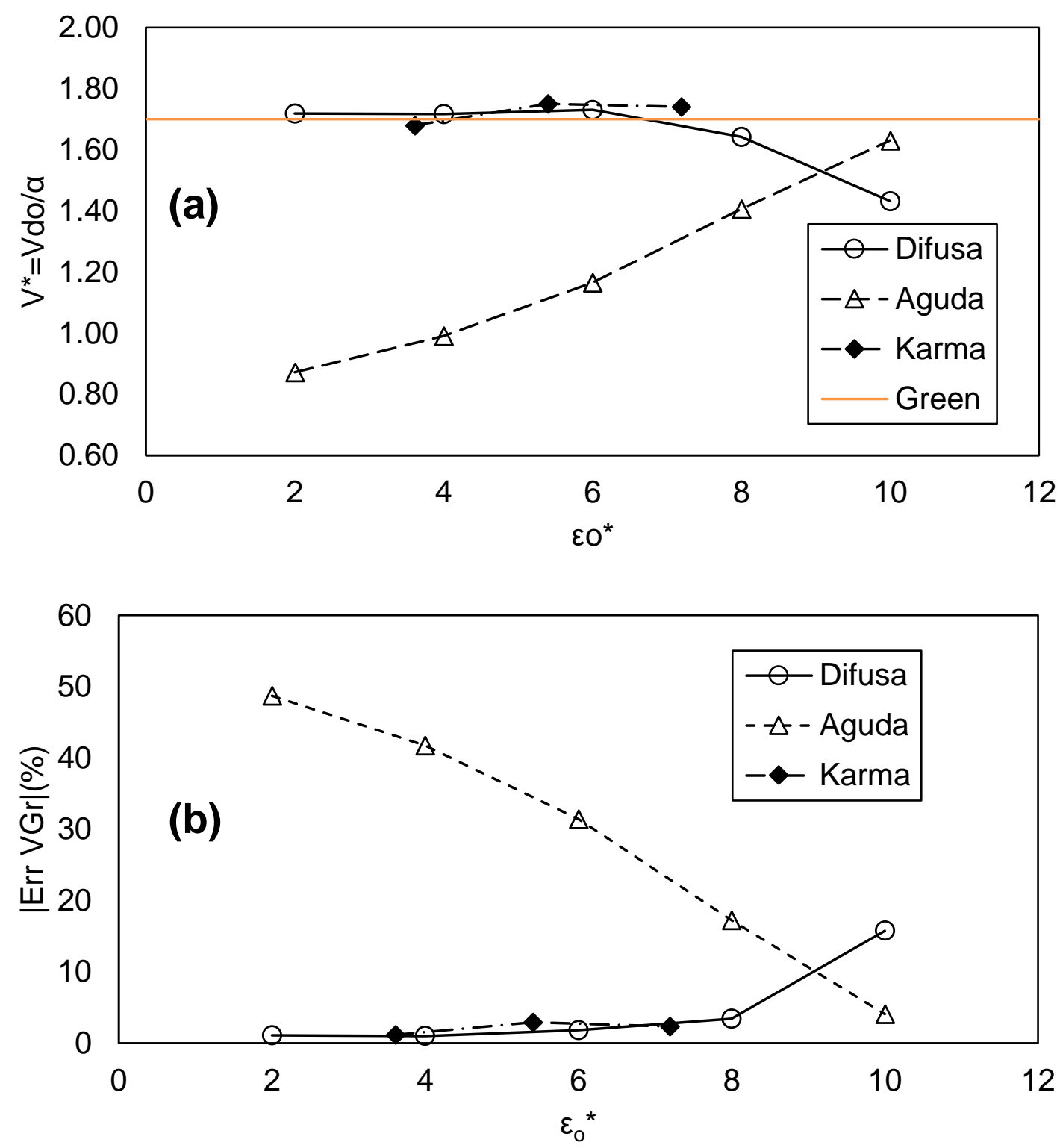

Figura 5 - Velocidade adimensional da ponta de dendrita após atingir estadoestacionário em função da espessura da interface difusa: (a) velocidade (b) erro em relação ao modelo clássico. $(\Delta=-0,55, s=0,05)$. Os cálculos de Karma e Rappel

[1] também estão apresentados em (a).

\section{CONCLUSÃo}

Os modelos de campo de fases de Wheeler, Boettinger e MacFadden (WBM) [2] e Karma e Rappel (KR) [1] foram implementados para estudar o efeito da espessura da interface sólido-líquido difusa na solidificação equiaxial de uma dendrita de metal puro. As imagens das dendritas calculadas pelo modelo WBM não convergem, ou seja, sofrem alterações até a menor espessura utilizada, igual a duas vezes o comprimento de capilaridade. No entanto, as imagens do modelo KR não apresentam variações quando a espessura é igual ou menor a quatro vezes 0 comprimento de capilaridade. A velocidade da ponta dendrítica em função do tempo calculada pelos dois modelos inicialmente assume um valor relativamente elevado, decrescendo para um valor constante, indicando estado-estacionário. As curvas do 
modelo WBM sofrem alterações significativas para variações da espessura de interface até o menor valor utilizado, enquanto as curvas do modelo KR não sofrem mais alterações tanto em sua região transiente como na estacionária para espessuras menores ou iguais a quatro vezes o comprimento de capilaridade. A velocidade de estado-estacionário dada pelo modelo KR converge para a solução do modelo clássico à medida que a espessura da interface é reduzida, ao passo que a velocidade fornecida pelo modelo WBM distancia-se cada vez mais do modelo clássico.

\section{Agradecimentos}

Agradecemos à CAPES e ao CNPq (311206/2014-0) pelas bolsas concedidas.

\section{REFERÊNCIAS}

[1] A. Karma and W.-J. Rappel, "Quantitative phase-field modeling of dendritic growth in two and three dimensions," Phys. Rev. E, vol. 57, no. 4, pp. 43234349, 1998.

[2] Wheeler, Boettinger, and McFadden, "Phase-field model for isothermal phase transitions in binary alloys.," Phys. Rev. A, At. Mol. Opt. Phys., vol. 45, no. 10, pp. 7424-7439, May 1992.

[3] J. Bragard, A. Karma, Y. H. Lee, and M. Plapp, "Linking Phase-Field and Atomistic Simulations to Model Dendritic Solidification in Highly Undercooled Melts," Interface Sci., vol. 10, no. 2/3, pp. 121-136, Dec. 2001.

[4] S. M. Allen and J. W. Cahn, "A microscopic theory for antiphase boundary motion and its application to antiphase domain coarsening," Acta Metall., vol. 27, no. 6, pp. 1085-1095, 1979.

[5] W. J. Boettinger, J. A. Warren, C. Beckermann, and A. Karma, "Phase-Field Simulation of Solidification," Annu. Rev. Mater. Res., vol. 32, no. 1, pp. 163194, Aug. 2002. 